\title{
Itch, Revisited
}

\author{
Mr. Ahmad AlAbdulkareem* \\ Royal College of Surgeons in Ireland 123, St Stephen's Green, Dublin 2, Dublin, Republic of Ireland
}

*Corresponding Author: Mr. Ahmad AlAbdulkareem, Royal College of Surgeons in Ireland 123, St Stephen's Green, Dublin 2, Dublin, Republic of Ireland, Email: Ahmadalabdulkareem@rcsi.ie

\begin{abstract}
Itch is the most frequent symptom reported in dermatology and is also commonly seen with other chronic clinical conditions. There is much overlap between itch and pain and recent work has elucidated some of the molecular and neuronal underpinnings of the itch-scratch response. Based on the cause, clinical itch is classified into: systemic, dermatologic, neuropathic or psychogenic. Regardless of the type, itch perception remains subjective and influenced by individual factors including cognition, reward, and emotion. This review aims to shed light on what has been established in terms of the molecular underpinnings of itch and the complexity of the itch-scratch response.
\end{abstract}

Keywords: Itch, scratch, pain, pruritus

\section{INTRODUCTION}

Itch (pruritus) has been defined as an "unpleasant sensation that elicits the desire or reflex to scratch". German physician Samuel Hafenreffer first described itch about 350 years ago [1], and while it sounds too familiar it hardly reflects the magnitude of the burden of itch. Indeed, itch is the most frequent symptom reported in dermatology and is also commonly seen with other chronic clinical conditions. Literature reports that more than 1 in 5 people will suffer from chronic itch at some point in their lifetime [2]. Itch can either be generated acutely as a protective response and is therefore transient, or become chronic and potentially debilitating. Due to the subjective and fluctuating nature of itch, and the difficulty in consistently comparing available data, itch research is still in its infancy. This review aims to shed light on what has been established in terms of the molecular underpinnings of itch and the complexity of the itch-scratch response.

\section{ITCH, OR OUCH?}

Despite the notion that pain is often associated with suffering and is therefore more emotionally provocative than itch, Itch (pruriception) has long been considered a submodality of pain (nociception). Both sensations are considered to be evolutionary protective responses. While itch triggers a scratch, pain elicits a withdrawal response. Several theories have also been proposed in an attempt to explain how pain relates to itch and the mechanisms by which pain neuronal pathways overlap with and/or inhibit those of itch.

The first of these theories was the intensity theory which proposed that the same population of peripheral nerves respond to both painful and itchy stimuli but at different intensity levels; at a lower threshold nerves transmit itch while the same stimulus at a higher intensity causes a transition to the perception of pain. This however was not proven to be the case as a low intensity painful stimulus does not necessarily trigger an itch [3].

The labeled line theory described two sets of separate neurons which respond to either itchy or painful stimuli. This came after the discovery of histamine-sensitive c nerve fibers which were unresponsive to mechanical stimuli and were distinct from other previously known nociceptors (pain-sensing neurons)3. However, data argued against the itch-only nature of these histamine-sensitive $c$ nerve fibers after it has been shown that they respond to capsaicin, a painful stimulus. Moreover, the experimental ablation of a subset of nociceptors led to significant reduction in scratch behavior further proving the intertwined nature of neurons encoding itch (pruriceptors) with those transmitting pain $[3,4]$. Evidence shows that candidate pruriceptors can often respond to 
painful stimuli, and known itchy stimuli (pruritogens) like histamine, cowhage and BAM8-22also induce a degree of pain, like stinging or pricking although itch sensations prevail $[5,6]$. On the other hand, it has also been documented the presence of peripheral nociceptors that do not respond to itchy stimuli. This led to the proposition of what is known as the population or selectivity theory. This theory suggests that pruriceptors are a subgroup within a larger population of nociceptors but are more selective and responsive to itch. Upon selective stimulation, pruriceptors encode itch but when a stimulus activates the larger nociceptor population, itch is masked by pain via inhibitory spinal pain-sensing neurons. However, if the stimulus was not strong enough, then mixed itch-pain sensations are produced [7].In support of the selectivity theory, studies have identified a group of peripheral neurons with an itchresponsive subset $[8,9]$. Centrally, capsiasin-a painful stimulus- has been shown to activate most neurons in the pain-sensing spinothalamic tract (STT) including neurons selective for pruritogens like histamine or serotonin $[10,11]$. Furthermore, the selective ablation of pruriceptors responsive to serotonin in rats led to pain-sensing defects, further proving the contribution of pruriceptors in encoding pain [12-14]. Lastly, scratching (pain) has been shown to inhibit itch-selective STT neurons in different animal models [15]. This model has its strength in demonstrating the presence of a specific itch neural pathway while accounting for the overlap with pain and the occasional antagonistic action of both. It, therefore, supports the idea that itch is not a submodality of pain but is rather a distinct form of sensation that happens to share many commonalities with pain.

\section{ITCH: THE CELlS, THE MoleculeS}

Historically, humans served as animal models of choice to investigate pruritus clinically. This, however, meant that molecular studies were not possible. After the development of animal models mimicking conditions where itch is commonly seen, the investigation of the molecular underpinnings of itch blossomed. Despite the subjectivity of parameters used, namely, scratch response assays, several molecules and their corresponding receptors have been identified as vital for the itch circuit. Peripheral free nerve endings have receptors that bind to pruritogens either directly or after their release from nearby cells. The signal is then transmitted via the aforementioned unmyelinated itch-sensitivec nerve fibers through the spinothalamic tract to be processed centrally [3].

Broadly, the itch response may either be histaminergic or nonhistaminergic. In the histaminergic response, histamine binds to $\mathrm{H} 1$ and/or $\mathrm{H} 4$ receptors both of which are G-protein coupled receptors to trigger itch. While histamine is the most investigated pruritogen and is often used in studies, the use of antihistamines clinically may often be ineffective highlighting the importance of other histamineindependent pathways. Mucunain, the main itchy stimulus isolated from cowhage, elicits strong pruritus without the wheal and flare response seen with histamine and is therefore an example of a nonhistaminergic itch. Similarly, other pruritogens include serotonin which binds to its 5-HT receptors and endothelin which in normally produced by endothelial cells but has been shown to trigger a burning itch upon intradermal injection. Han et al. summarized established pruritogens and their corresponding receptors in table 1 [16].

Table1: Pruritogens and receptors in the periphery

\begin{tabular}{|l|l|l|}
\hline \multicolumn{1}{|c|}{ Pruritogen } & \multicolumn{1}{|c|}{ Source(s) } & \multicolumn{1}{|c|}{ Receptor(s) in DRG } \\
\hline Histamine & Mast cells & $\mathrm{H} 1 \mathrm{R}, \mathrm{H} 4 \mathrm{R}$ \\
\hline BAM8-22 & Proteolytic cleavage of proenkephalin & MrgprC11 \\
\hline CQ & Antimalaria medicine & MrgprA3 \\
\hline SLIGRL-NH 2 & $\begin{array}{l}\text { Synthetic peptide or proteolytic cleavage of } \\
\text { PAR2 }\end{array}$ & MrgprC11, PAR2 \\
\hline$\beta$-alanine & Muscle-building supplement & MrgprD \\
\hline Mucunain & Cowhage & PAR2, PAR4 \\
\hline Cathepsin S & Immune cells and epithelial cells & PAR2, PAR4 \\
\hline Tryptase & Mast cells & PAR2, PAR4 \\
\hline 5-HT & Mast cells & 5-HT1, 5-HT2 \\
\hline ET-1 & Endothelial cells & ET \\
\hline Substance P & Primary sensory neurons & NK1 \\
\hline
\end{tabular}




\begin{tabular}{|l|l|l|}
\hline IL-31 & Mainly Th2 lymphocytes & IL-31RA, OSMR \\
\hline Imiquimod & Synthetic immunostimulant & TLR7 (controversial) \\
\hline Polyinosinic:polycytidylic acid & Synthetic immunostimulant & TLR3 \\
\hline Bile acid & Cholestasis & TGR5 \\
\hline Lysophosphatidic acid & Cholestasis & Unknown \\
\hline
\end{tabular}

Abbreviations: BAM8-22, bovine adrenal medulla 8-22 peptide; CQ, chloroquine; DRG, dorsal root ganglion; ET-1, endothelin-1; IL-31, interleukin-31; 5-HT, serotonin.

There has been attempts to investigate the role of these molecular candidates in pathologies related to chronic itch. Rodent models overexpressing the endogenous pruritogen IL-31 have been shown to experience severe scratch resulting in lesions that mimic atopic dermatitis [17]. Likewise, patients with atopic dermatitis have elevated serum levels of Substance P and Tryptase, and increased expression of pruritogen-receptor PAR2 [18, 19].

While much has been uncovered in terms of the molecular fundamentals of acute itch, most are peripheral mediators and molecules involved centrally -at the level of the spinal cord- are yet to be explored. Also, some cells are known to be heavily involved in the itch circuit including mast cells and keratinocytes. However, the complex interplay between the different cells types including immune cells is not as well understood. It is also worth to mention that altering animal models to investigate the role of certain mediators of pathological itch may not yield conclusive results in patients, where the line between pathological itch and pain gets blurred.

\section{A PURE ITCH: Does IT EXIST?}

According to the most accepted itch hypothesis, the selectivity theory, pruriceptors that encode for itch are a subgroup within the larger population of pain-sensing nociceptors. Pruriceptors respond preferentially yet not exclusively to pruritogens which can be endogenously produced or exogenously applied and/or administered.

As aforementioned, many known pruritogens including histamine, cowhage and BAM8-22 trigger itch-scratch responses accompanied by sensations of stinging, pricking or burning. While these sensations may not necessarily be painful, they are nevertheless examples of nociception. Despite the fact that those overlapping sensations may be seen in favor of classifying itch as a minor form of pain, everyday life is a clear demonstration that a pure itch sensation without pain-associated components exists and can be triggered mechanically. Contact with wool fibers triggers a pure itch with no flare or mixed sensations; this supports the idea that mechanosensitive pruriceptors of a tactile nature may exist and are therefore distinct from other pruriceptors previously explained by the selectivity theory. Recently, a group managed to successfully reproduce this pure mechanically-evoked itch experimentally [20]. They used a vibrating stainless-steel wire loop at a specific range and frequency to apply a mechanical stimulus on human villus hair. This produced an itch with no flare and which was not responsive to antihistamine therapy. Upon continuous stimulation, the itch gradually weakened and was not felt after about 10 minutes. The observed features of low-activating thresholds, intermediate adaptation, and fatigue after repeated stimulation support the possibility of ctactile neuron involvement. However, further microneurographic studies are needed to better elucidate this seemingly separate itch neural pathway.

\section{Clinical ITCH}

\subsection{Systemic Itch}

Many systemic conditions have the symptom of itch as a prominent and often debilitating feature. In fact, chronic itch may be considered comparable to if not worse than chronic pain for which medications are arguably more effective. For example, the vast majority of patients with cholestatic liver diseases suffer from chronic pruritus [21]. Occasionally, those patients may even be considered for a liver transplant solely on the basis of how debilitating their itch is and its impact on quality of life. It has been proposed that bile acid is the main pruritogen in those patients since serum level of bile acids is elevated due to the impaired liver secretion. Experiments have also demonstrated that direct application of bile acid on human skin triggers itch [22]. Furthermore, the use of bile sequestering agents like cholestyramine relieves itch clinically21. Recently, lysophosphatidic acid (LPA) has emerged asanother possible candidate in mediating itch, and the evidence supporting it comes from the discrepancy in LPA levels between pruritic patients and patients not reporting itch [23]. Further studies are still required since the exact pathogenesis of 
cholestatic itch is still largely unknown and treatment strategies can be ineffective.

Uremicpruritus is another example of systemic itch and is a major cause of morbidity in patients with renal dysfunction. Itch is also common in oncological settings. It can be caused by an underlying cancer like leukemia or a lymphoma. It can also be a feature suggestive of hepatic or renal invasion, or a side effect of medications used. Itch can also accompany endocrine conditions like hyperparathyroidism and thyrotoxicosis, and in deficiency states like in iron deficiency [24].

\subsection{Dermatologic Itch}

Itch is possibly the single commonest symptom for skin disorders. Dermatologic itch often causes localized itchy rashes unlike neuropathic itch where they may be no rashes or apparent skin lesions. Identifying such rashes is a clinical challenge for which a proper thorough history is key. This would allow for the differentiation of primary diseased skin triggering itch from secondary scratch lesions. Examples of dermatologic conditions causing such itch include: Atopic dermatitis, eczema, lichen sclerosis, and skin infections like lice, candidiasis and other fungal infections [25].

\subsection{Neuropathic Itch}

By definition, neuropathic itch is the perception of itch despite the absence of an itchy stimulus. It can be thought of as equivalent to other forms of sensory hallucinations. It often arises due to dysfunctional or diseased neurons and can be seen in many neurologic conditions including tumors, peripheral neuropathies and multiple sclerosis. Little is known about the molecular pathophysiology underpinning neuropathic itch and most available evidence has been accumulated by studying neuropathic pain. Clinically, neuropathic itch is usually only considered after excluding possible systemic or dermatologic causes [25].

\subsection{Psychogenic Itch}

Itch is a complex and a multidimensional somatosensory sensation. The itch-scratch behavior is therefore subjective and involves discriminative, cognitive, and emotional and reward components. This becomes evident in psychogenic itch, known clinically as somatoform pruritus where no real stimulus is identified. Psychogenic itch can be seen in patients with other psychiatric and psychosomatic co-morbidities including schizophrenia and obsessive compulsive disorders. Those patients often have to be managed with psychotherapy and possibly antidepressants [16].

Historically, doctors may have been guilty of interpreting clinically-unexplained signs and symptoms including itch as psychogenic. Likewise, patients may also harbor illogical explanations for their chronic itch. These may include infestation with an undiagnosed parasite; a condition termed primary delusional parasitosis [25]. Other patients may have strong beliefs that there are tiny fibers or threads in or on their skin. These explanations may stem from social, cultural or traditional beliefs, further challenging clinicians to find plausible and actionable reasons.

Psychogenic itch is also commonly seen in healthy subjects. Contagious itch, for example, can be easily triggered by visual cues. Literature shows that most healthy people scratch empathetically after watching videos of other people itching and that is considered to be a normal social response [16]. This supports the subjectivity of the scratch behavior and the fact that it is influenced by psychosocial factors regardless of whether sensory stimuli are present.

\section{GAPS TO BE Filled}

Recently, rapid progress has been made in itch research. Many molecular candidates have been identified, theories have been proposed, and many attempts to explain the exact pathophysiological mechanisms and circuits of itch have been made. Despite the efforts, key questions remain unsolved. Better animal models are needed to better understand clinical itch and how it can be optimally managed. At a molecular level and as mentioned earlier, while molecules and their corresponding receptors have been identified, most are peripheral and central mediators are still largely unknown. Likewise, central elements of the itch circuit including the thalamus and cortex and their connections are not well established and the role of spinal interneurons and the complex interplay of cells need to be investigated in greater detail. Further studies on mediators of systemic itch are also required given their potential as therapeutic targets for such a widely-prevalent and debilitating complaint. Lastly, will the selectivity theory be the last of the theories despite it being at variance with the everyday observation of mechanically-evoked itch sensations? 


\section{CONCLUSION}

Itch is a widely-recognizable complaint by many patients and is also commonly observed in everyday life. There is much overlap between itch and pain yetexperiments have proved itch to be a distinct modality of sensation. Recent work has elucidated some of the molecular and neuronal underpinnings of the itch-scratch response, although a more detailed account is still needed. Based on the cause, clinical itch is classified into: systemic, dermatologic, neuropathic or psychogenic. The perception of itch is subjectivesince it is influenced by individual factors including cognition, reward, and emotion. Indeed, the Buddhist philosopher Nāgārjuna appropriately stated, "There is pleasure when an itch is scratched. But to be without an itch is more pleasurable still."[26].

\section{REFERENCES}

[1] Ikoma A, Steinhoff M, et al. The neurobiology of itch. Nat Rev Neurosci. 2006 Jul; 7(7):5354 7

[2] Weisshaar E, Matterne U. Epidemiology of Itch. In: Carstens E, Akiyama T, editors. Itch: Mechanisms and Treatment. Boca Raton (FL): CRC Press/Taylor \& Francis; 2014. Chapter Available from: https://www.ncbi.nl m.nih.gov/ books/NBK200924/

[3] McMahon SB, Koltzenburg M.Itching for an explanation.Trends Neurosci. 1992 Dec; 15(12):497-501.

[4] Imamachi N, Park GH, Lee H, et al. TRPV1expressing primary afferents generate behavioral responses to pruritogens via multiple mechanisms. Proceedings of the National Academy of Sciences of the United States of America. 2009; 106(27):11330-11335. doi: 10.1073/pnas.0905605106. Available from: http://www.pnas.org/content/106/27/11330.full

[5] Reddy VB, Iuga AO, et al. Cowhage evoked itch is mediated by a novel cysteine protease - a ligand of protease activated receptors. The Journal of neuroscience: the official journal of the Society for Neuroscience. 2008; 28(17): 4331-4335. Doi: 10.1523/ JNEUR OSCI.071608.2008. Available from: http:// www. jneurosci. org/content/28/17

[6] Sikand P, Dong X, LaMotte RH. BAM8-22 peptide produces itch and nociceptive sensations in humans independent of histamine release. The Journal of neuroscience: the official journal of the Society for Neuroscience. 2011; 31(20):7563-7567. Doi: 10.1523/JNEUR OSCI.1192-11.2011. Available from: http:// www. jneurosci.org/content/31/20/7563

[7] Patel KN, Dong X. An Itch To Be Scratched. Neuron. 2010; 68(3):334-339. Doi: 10.1016/ j.neuron.2010.10.018. Available from: http://
www.cell.com/neuron/fulltext/S0896-6273(10) 00868-8

[8] Lagerström MC, Rogoz K, Abrahamsen B, et al. VGLUT2-Dependent Sensory Neurons in the TRPV1 Population Regulate Pain and Itch. Neuron. 2010; 68(3):529-542. Doi: 10.1016/j. neuron.2010.09.016. Available from: https:// www.sciencedirect.com/science/article/pii/S08 96627310007580

[9] Liu Y, Samad OA, Zhang L, et al. VGLUT2dependent Glutamate Release from Nociceptors Is Required to Sense Pain and Suppress Itch. Neuron. 2010; 68(3):543-556. Doi:10.1016/j. neuron.2010.09.008. Available from: http:// www.cell.com/neuron/pdf/S0896-6273(10)00 725-7.pdf

[10] DA S, Zhang X, et al. Comparison of responses of primate spinothalamic tract neurons to pruritic and algogenic stimuli.J Neurophysiol. 2004 Jan; 91(1):213-22.

[11] Jinks SL, Carstens E. Responses of superficial dorsal horn neurons to intradermal serotonin and other irritants: comparison with scratching behavior. J Neurophysiol. 2002 Mar; 87(3):1280 9.

[12] Carstens EE, Carstens MI, et al. Dorsal horn neurons expressing NK-1 receptors mediate scratching in rats. Neuroreport. 2010 Mar 10; 21(4):303-8. Available from: http://pubmed centralcanada.ca/pmcc/ articles/PMC3123731/

[13] PW Mantyh, SD Rogers, et al. Inhibition of hyperalgesia by ablation of lamina I spinal neurons expressing the substance $P$ receptor.Science. 1997 Oct 10; 278(5336):275-9.

[14] ML Nicholas, BJ Allen, et al. Transmission of chronic nociception by spinal neurons expressing the substance $\mathrm{P}$ receptor. Science. 1999 Nov 19; 286(5444):1558-61.

[15] Davidson S, Zhang X, et al. Relief of itch by scratching: state-dependent inhibition of primate spinothalamic tract neurons. Nature neuroscience. 2009; 12(5):544-546. doi:10.10 38/nn.2292.

[16] Han L, Dong X. Itch Mechanisms and Circuits. Annual review of biophysics. 2014; 43:331355. Doi: 10.1146/annurev-biophys-051013022826. Available from: http://europepmc. org/articles/PMC408 1479

[17] Dillon SR, Sprecher C, et al. Interleukin 31, a cytokine produced by activated $\mathrm{T}$ cells, induces dermatitis in mice. Nat. Immunol. 2004; 5:75260. Available from: http://www.noar.technion. ac.il/images/attachments/Scitech/2016/ Interleu kin $\% 2031, \% 20 \mathrm{a} \% 20$ cytokine $\% 20$ produced $\%$ 20by\%20activated.pdf

[18] Toyoda M, Nakamura M, et al. Nerve growth factor and substance $\mathrm{P}$ are useful plasma 
markers of disease activity in atopic dermatitis. Br. J. Dermatol. 2002; 147:71-79.

[19] Sujii K, Andoh T, et al. Involvement of tryptase and proteinase-activated receptor- 2 in spontaneous itch-associated response in mice with atopy-like dermatitis. J. Pharmacol. Sci. 2009; 109:388-95.

[20] Fukuoka M, Miyachi Y, Ikoma A. Mechanically evoked itch in humans. Pain. 2013; 154:897-904.

[21] Bunchorntavakul C, Reddy KR. Pruritus in chronic cholestatic liver disease. Clin. Liver Dis. 2012; 16:331-46.

[22] Varadi DP. Pruritus induced by crude bile and purified bile acids. Arch. Dermatol. 1974; 109:678-81.

[23] Kremer AE, Martens JJ, Kulik W, Rueff F, Kuiper EM, et al. Lysophosphatidic acid is a potential mediator of cholestatic pruritus.
Gastroenterology. 2010; 139:1008-18.e1. Available from: http://www.icpsupport. org/ pdf/Kremer.pdf

[24] Ständer S, Weisshaar E, et al. Clinical classification of itch: A position paper of the International Forum for the Study of Itch. ActaDerm. Venereol. 2007; 87((4)):291-294. Available from: https://www.itchforum. Net/PDFs/ 26971.pdf

[25] Oaklander AL. Neuropathic Itch. In: Carstens E, Akiyama T, editors. Itch: Mechanisms and Treatment. Boca Raton (FL): CRC Press/Taylor \& Francis; 2014. Chapter 7. Available from: https://www. ncbi.nlm.nih.gov/books/NBK200 940/

[26] Bautista DM, Wilson SR, Hoon MA. Why we scratch an itch: the molecules, cells and circuits of itch. Nature neuroscience. 2014; 17(2):175182. doi:10.1038/nn.3619. Available from: http://europepmc.org/ articles /PMC4364402

Citation: Mr. Ahmad AlAbdulkareem Itch, Revisited, ARC Journal of Dermatology. 2019; 4(2):7-12. doi:dx.doi.org/10.20431/2456-0022.0402003.

Copyright: (C) 2019 Authors. Thisisan open-access article distributed under the terms of the Creative Commons Attribution License, which permits unrestricted use, distribution, and reproduction in any medium, provided the original author and source are credited. 